\title{
La plataforma YouTube como estrategia para el autoaprendizaje de la lengua inglesa
}

\section{The YouTube website as a strategy for self-learning of the English language}

Rodrigo Ruay G'.

\section{Elvis Campos $\mathrm{P}^{2}$}

Universidad de La Serena La Serena - Chile

\section{RESUMEN}

Los autores presentan un estado de avance de un proyecto de investigación pedagógica aplicada donde se utiliza la red social YouTube

$1 \quad$ Investigador del Instituto Multidisciplinario de Ciencia y Tecnología y Académico de la Unidad de Gestión Integrada de las Pedagogías de La Universidad de la Serena.rruay@userena.cl Telefono 512204000

Universidad de La Serena La Serena - Chile

RODRIGO ORLANDO RUAY: https://orcid.org/0000-0003$\underline{3283-4651}$

2 Director de Escuela de Humanidades, Coordinador y Académico de la Carrera de Pedagogía en Inglés de la Universidad de La Serena.

Elvis Campos P. ecampos@userena.cl

Teléfono 512204000

Universidad de La Serena La Serena - Chile campos https://orcid.org/0000-0003-1631-0495 como herramienta de aprendizaje autónomo para el desarrollo de las habilidades de la lengua inglesa. Esta tiene como propósito verificar si la exposición metódica y autorregulada a un canal de YouTube incide favorablemente en el desarrollo de habilidades del idioma inglés en estudiantes de enseñanza media y universitarios de la Carrera Pedagogía en inglés, de una Universidad pública regional de Chile.

La metodología empleada para esta investigación fue el estudio de casos múltiples, haciendo uso de un sitio Web liberado como lo es YouTube, siguiendo canales de tipo académico 
dirigidos por profesores de habla inglesa. Estos contienen lecciones del idioma, que incluyen aspectos de la lengua, tales como: gramática, fonética, vocabulario, expresiones formales e informales. Entre los principales hallazgos que se pueden relevar están: la motivación, el interés, valoración del aprendizaje del idioma inglés y el aprovechamiento del tiempo libre para desarrollar aprendizaje autónomo. Esto se evidencia en la elaboración de fichas didácticas, estrategia con la cual registraron y sistematizaron la información contenida en cada clase del canal seleccionado, como una herramienta de autoaprendizaje.

Palabras clave. Tiempo libre, aprendizaje virtual, autonomía educativa, aprendizaje autónomo, habilidades de la lengua inglesa.

\section{ABSTRACT}

The authors present a state of progress of an applied pedagogical research project where the YouTube social network is used as an autonomous learning tool for the development of English language skills. This is intended to verify whether the methodical and self-regulated exposure to a YouTube channel has a favorable impact on the development of English language skills in middle school and university students of the English Pedagogy Program, of a regional public University of Chile

The methodology used for this research was the study of multiple cases, using a website released such as YouTube, following academic channels directed by English-speaking teachers. These contain language lessons, which include aspects of the language, such as: grammar, phonetics, vocabulary, formal and informal expressions. Among the main findings that can be highlighted are: motivation, interest, assessment of English language learning and the use of free time to develop autonomous learning. This is evidenced in the elaboration of didactic files, strategy with which they registered and systematized the information contained in each class of the selected channel, as a self-learning tool.

Keywords. Free time, virtual learning, educational autonomy, autonomous learning, English language skills.

\section{INTRODUCCIÓN}

Los programas formales de asignaturas contienen un apartado llamado estrategias metodológicas, donde se registran métodos tales como: clase magistral, estudio de casos, resolución de problemas, aprendizaje por proyectos, contratos de aprendizajes y otros. En general, el uso de las TIC como estrategia metodológica para el aprendizaje es incipiente, y en particular en la plataforma YouTube como un recurso de aprendizaje para la lengua inglesa.

YouTube es un portal Web, ampliamente conocido por los usuarios de Internet, provee el servicio de compartir videos de las más diversas índoles, de manera que se puede convertir, si los profesores lo desean, en una herramienta fundamental a la hora de reforzar contenidos que quizás no se comprendieron en clases o para aprender cosas nuevas que complementen un saber previo. También es posible llegar a un aprendizaje totalmente nuevo, tanto para estudiantes del sistema educativo, como para cualquier persona que esté interesada en aprender algo específico de la lengua extranjera, en este caso inglés.

El estudio es abordado desde dos grandes áreas, por una parte, el uso de la plataforma como una herramienta didáctica para el desarrollo de las habilidades de la lengua inglesa, teniendo como referente un enfoque comunicativo y el Marco Común Europeo de Referencia para las Lenguas (MCER., 2001), y por otra el enfoque de aprendizaje y trabajo autónomo del estudiante.

En cuanto a enfoques de enseñanza de la lengua, el método comunicativo es más que un sistema de hábitos que pueden ser formados a 
través de una ejercitación mecánica. La lengua es un sistema específico y el aprendiz debe saber cómo este sistema acciona la comunicación real como medio para lograr un fin" (Hernández, 2000, p. 148)

El enfoque comunicativo o nocional-funcional pretende capacitar al alumnado para la comunicación real. En la versión débil se integran actividades que den la oportunidad a los alumnos de usar la L2 con fines comunicativos en un programa más amplio haciendo que se practique lo aprendido. En la fuerte se adquiere la lengua directamente hablándola y comunicándose, y no supone una activación de lo aprendido sino la fuente de aprendizaje en sí (Howatt, 1985:279).

El enfoque comunicativo comparte ciertas características del conductismo, una alta dosis del cognitivismo y del humanismo, integra lo funcional y lo gramatical, se preocupa por tanto de los aspectos funcionales de la lengua como de los estructurales. Sobresalen aspectos como: la importancia del significado, los elementos son presentados en un contexto, aprender la lengua es aprender a comunicarse, busca desarrollar la habilidad para usar el sistema lingüístico de manera efectiva y apropiada, el objetivo fundamental es la fluidez y el dominio aceptable de la lengua. La meta de la enseñanza de una lengua es desarrollar lo que Hymes (1972) señaló como la competencia comunicativa.

El estudio incorpora las orientaciones del Marco Común Europeo de Referencia para las Lenguas (MCER) porque promueve un enfoque orientado a la acción. Lo denomina así dado que considera a los usuarios y alumnos que aprenden una lengua como agentes sociales, como miembros de una sociedad que tiene tareas que llevar a cabo, en una serie determinada de circunstancias, en un entorno específico y dentro de un campo de acción concreto.
La otra área abordada en esta investigación es el aprendizaje autónomo y el aprovechamiento del tiempo libre eficiente para un trabajo autónomo. En este contexto, es preciso señalar que el ejercicio de la autonomía del estudiante consiste en la posibilidad de aprender el idioma inglés de modo personalizado, haciendo uso de sus propios recursos y estrategias de aprendizaje.

Este enfoque basado en la acción, también tiene en cuenta los recursos cognitivos, emocionales y volitivos de la persona, así como toda la serie de capacidades específicas que el alumno aplica como agente social, estos son componentes claves que subyacen en forma explícita e implícita en el curriculum de la formación inicial de profesores. De esta manera, cualquier acto de aprendizaje o de enseñanza de idiomas, estaría de alguna manera, relacionada con una serie de dimensiones, tales como: las estrategias, las tareas, los textos, las competencias generales de un individuo, la competencia comunicativa, los procesos, los contextos y los ámbitos.

La otra área abordada en esta investigación es el aprendizaje autónomo del estudiante y el aprovechamiento eficiente de las horas de trabajo autónomo establecidas en la asignatura.

Se busca estimular la confianza del estudiante en sus propias capacidades y en sus conocimientos previos, su capacidad de aprender sin depender en todo momento del libro y del profesor (Ruay y Garcés, 2015). Tal independencia y autonomía se concretan fundamentalmente en el uso de fichas de registro de lo observado en la Web. Se concreta también en ejercicios específicos relacionados con la programación, planificación y autoevaluación de las actividades de aprendizaje y de las técnicas empleadas; finalmente, la independencia y autonomía se concretan en el carácter abierto y opcional de las actividades.

El trabajo autónomo del estudiante ha de ser un espacio para desarrollar el aprendizaje personal 
e independiente como condición básica para continuar aprendiendo durante toda la vida, si bien se plantea dentro de los ideales formativos y dentro de los perfiles de los educandos, no parece haber tenido un espacio propio en los currículos. Pareciera ser que es un aprendizaje que se da por supuesto o por añadidura, que se le traslada a escenarios no formales o no escolares, y muchas veces se le reduce a un conjunto de técnicas de estudio.

\section{DESCRIPCIÓN Y FUNDAMENTACIÓN DEL ESTUDIO}

Como se señaló anteriormente la investigación se aboca a indagar como se desarrollan las habilidades de la lengua inglesa, teniendo a la base el enfoque del método comunicativo. En este método, se pueden distinguir dos etapas, una clásica y una contemporánea, pero en ambos se definieron componentes básicos en la enseñanza. Estos son: un contexto, donde se lleva a cabo la comunicación, un rol que asume cada interlocutor, una función del lenguaje que se utiliza en cada situación comunicativa, nociones, conceptos que el estudiante necesita para llevar a cabo la comunicación, habilidades discursivas a desarrollar para conectar el discurso y hablar en forma coherente, un contenido gramatical y un contenido léxico.

Con estos elementos se propusieron nuevos formatos de currículo o programas de estudio. En primer lugar, nació el currículo basado en habilidades. Este currículo parte de las cuatro habilidades de la lengua y las desglosa en sub-habilidades. Por ejemplo, la habilidad de escuchar que una macro habilidad, puede ser desglosada en micro habilidades, tales como: reconocer palabras claves, reconocer el tema de la conversación, reconocer la actitud del hablante hacia el tema de conversación, reconocer referencias de tiempo, mantener la atención a pesar de distintas velocidades en el habla. (Richards y Rodgers, 1986)
En este enfoque se parte del supuesto que el alumno trae al aula su propia idea de cómo debería ser la enseñanza-aprendizaje. Se relacionan más entre ellos que con el profesor, existe poca corrección de errores, cooperan unos con otros en el proceso de aprendizaje. El rol que juega el profesor es de facilitador del proceso de comunicación entre los alumnos, las actividades y los textos. Actúa como participante del grupo, organiza recursos, guía los procedimientos y actividades de la clase, contribuye con su conocimiento, habilidades y experiencia al aprendizaje y organización de la clase. Analiza las necesidades y es gestor del proceso del grupo, entre otros.

En el desarrollo de las actividades de aprendizaje se utilizan una variedad de recursos, siendo estos de tres tipos:

- centrados en el texto, libros con el cual se desarrollan tareas planteadas y se analizan mediante ejercicios prácticos.

- Centrados en la tarea, se utilizan una gran variedad de juegos, simulaciones y actividades comunicativas tanto grupal como personalizado.

- materiales auténticos, uso de materiales no adaptados y reales: señales, revistas, anuncios, periódicos, mapas, dibujos, sitios webs, entre otros. (Richards y Rodgers, 1986)

En consideración a lo anteriormente expuesto, el Marco Común Europeo de Referencia para las Lenguas (MCER, 2002) orienta la metodología comunicativa hacia una toma en consideración del aprendiz como un sujeto del aprendizaje que implica el desarrollo de la capacidad de aprender a aprender, el desarrollo de la capacidad de autoevaluación, el desarrollo de una capacidad de autoevaluación y el desarrollo de una competencia estratégica y metacognitiva. 
En la enseñanza de segundas lenguas había sido habitual enseñar por separado las cuatro destrezas lingüísticas: comprensión auditiva, expresión oral, comprensión lectora y expresión escrita. Aun cuando en la vida real lo más habitual es que las distintas destrezas se combinan entre sí. De tal modo que en la segunda mitad del siglo XX se empezó a considerar la integración de las destrezas, lo cual se entiende como la combinación de dos o más habilidades entrelazadas a semejanza de la vida real y a diferencia de algunos modelos didácticos como el audio lingüista.

EI MCER establece una tipología de actividades comunicativas que, además de recoger las cuatro destrezas clásicas, incluye nuevas perspectivas y conceptos; los cuatro tipos de resultantes son: comprensión, expresión, interacción y mediación.

Cuando se aprende una lengua para comunicarse y expresarse en ella, se trata de llevar a cabo actividades comunicativas (escuchar noticias, escribir una carta, tomar la palabra, etc.), para cumplir funciones (dar y obtener información, expresar agrado, expresar simpatía, etc.), en determinadas situaciones socioculturales, con recursos lingüísticos (gramaticales, léxicos, discursivos, fonéticos, gráficos), adecuado a la situación de comunicación, activando procesos, estrategias y actitudes que posibilitan el aprendizaje y la comunicación (MCER, 2002).

Cada situación comunicativa supone una combinación de varios tipos de actividades, escuchar, leer, interactuar, redactar, hasta mediar para que otro alumno participe de la situación.

EI MCER ofrece un conjunto estructurado de descriptores para una amplia gama de actividades, organizadas en la siguientes siete categorías: expresión oral, expresión escrita, comprensión auditiva, comprensión de lectura, comprensión audiovisual, interacción oral e interacción escrita, y se añaden dos: mediación oral y mediación escrita.(MCER, 2002) Estas medicaciones, a juicio de los investigadores se presentan muy bien trabajadas en las actividades de aprendizaje modeladas en la plataforma YouTube.

Otro de los componentes claves que la investigación buscó relevar fue el aprovechamiento del tiempo libre para el trabajo y aprendizaje autónomo del estudiante, al respecto De Miguel (2006) señala que tanto el estudio y trabajo autónomo es una modalidad de aprendizaje en la que el estudiante se responsabiliza de la organización de su trabajo y de la adquisición de las diferentes competencias según su propio ritmo. Implica por parte de quien aprende asumir la responsabilidad y el control del proceso personal de aprendizaje, y las decisiones sobre la planificación, realización y evaluación de la experiencia de aprendizaje.

El aprendizaje autónomo, en estudiantes de educación superior, parece estar constituido (Pintrich y Groot, 1990; Vermunt, 1995) por tres importantes aspectos: a) estrategias cognitivas o procedimientos intencionales que permiten al estudiante tomar las decisiones oportunas de cara a mejorar su estudio y rendimiento; b) estrategias metacognitivas o de reflexión sobre el propio proceso de aprendizaje y c) estrategias de apoyo referidas al autocontrol del esfuerzo y de la persistencia, y a promover condiciones que faciliten afectivamente el estudio. Así pues, el aprendizaje autónomo es un proceso que permite al estudiante ser autor de su propio desarrollo. La estimulación de este aprendizaje persigue, esencialmente, junto a la generación de espacios de libertad curricular, lograr en el estudiante, y en consecuencia en el futuro profesional, un grado de autonomía que lo habilite para su propio gobierno, el aprendizaje continuo, la toma de decisiones y la gestión independiente o vinculada a otros profesionales. Por ello es esencial que se tenga en cuenta: la 
contextualización de los aprendizajes, la reflexión personal, la construcción de conocimientos, la aplicación práctica de los mismos y la evaluación del proceso realizado.

A partir de lo anteriormente señalado, un aprendizaje entendido como construcción del sentido del conocimiento, en donde se privilegian los procesos por medio de los cuales el estudiante codifica, organiza, elabora, transforma e interpreta la información recogida. Supone un nivel de aprendizaje autónomo y estratégico (Pozo y Monereo, 1999) que consiste en saber utilizar las propias competencias y los recursos más adecuados a las condiciones contextuales en las que se debe actuar. El estudiante autónomo, como sujeto a cargo de su propio aprendizaje, se formula metas, organiza el conocimiento, construye significados, utiliza estrategias adecuadas y elige los momentos que El aprendizaje autónomo requiere un replanteamiento de los procesos cotidianos de enseñanza y aprendizaje, pero igualmente de una capacitación específica a los docentes sobre estrategias que promuevan el desarrollo de habilidades para el aprendizaje autónomo del estudiante, tales como la realización de mapas conceptuales y mapas mentales, el diseño de esquemas y la graficación de procesos, el aprendizaje basado en problemas (ABP), los estudios de casos, las matrices de resignificación de experiencias, el diseño de proyectos individuales y grupales, exposiciones orales ante los pares, participación en paneles y debates, simulación de roles en mesas de jueces entre otros.

El trabajo autónomo del estudiante abarca igualmente el manejo pedagógico de las TIC, el manejo de diferentes medios como las aulas virtuales con sus múltiples recursos (foros y chats, ejercicios, enlaces en Internet) y de las bases de datos electrónicas, y en general el manejo de materiales en red. considera pertinentes para adquirir, desarrollar y generalizar lo aprendido.
Según Crispin (2011), Algunas de las herramientas para promover aprendizaje autónomo en los estudiantes son: Internet abierto, revistas electrónicas y libros electrónicos, aulas virtuales, Google académico, portales con direcciones electrónicas, tutoriales online, clases espejo, Webinars, entre las más relevantes.

Para efectos de este estudio, el trabajo autónomo es un aprendizaje estratégico en el que el estudiante toma decisiones claves sobre su propio aprendizaje de la lengua inglesa: autodirigiéndolo en función de sus necesidades, metas o propósitos, auto regulándolo y autoevaluándose, de acuerdo con los recursos y escenarios de que dispone en la plataforma.

La consecuencia de su trabajo es que con el aprendizaje autónomo aprende a aprender gracias al entrenamiento y desarrollo de competencias o habilidades cognitivas, afectivas e interactivas, pero también, y de manera esencial, gracias al desarrollo de habilidades metacognitivas.

Considerando lo anteriormente expuesto la investigación se planteó el siguiente objetivo: Implementar un método para desarrollar el trabajo autónomo del estudiante de Tercer Año Medio y de Segundo año de la carrera de Pedagogía en Inglés de la Universidad de La Serena, utilizando un canal de YouTube, como herramienta de aprendizaje de las habilidades comunicativas de la lengua inglesa.

\section{METODOLOGÍA.}

Esta investigación se inscribe en un paradigma cualitativo con un diseño estudio de caso múltiple (Stake, 1999), en el entendido que el estudio de casos es el estudio de la particularidad y de la complejidad de un caso singular (Ruíz, J. 1996) para llegar a comprender su actividad en circunstancias importantes. 
La metodología de estudio de casos en este proyecto de investigación busca profundizar en unidades de análisis específicas (Tamayo y Tamayo, 2004), 07 estudiantes de Tercer año medio del Colegio Municipal José Manuel Balmaceda de la Serena y 11 estudiantes de segundo año de la carrera de Pedagogía en Inglés de la Universidad de La Serena. De ellos se recoge información de las actividades de aprendizaje que realizan con la plataforma YouTube.

Las técnicas de Recolección de datos de la Investigación son: Análisis documental, mediante sistematización de fichas de trabajo de los estudiantes como evidencia del uso de la plataforma YouTube; Focus Group donde se conversará respecto a dos ejes principales: la valoración del aprendizaje realizado con los canales YouTube y el trabajo autónomo. En el primer eje cada participante relatará la razón que lo llevó a adoptar un canal específico, describir la metodología utilizada por el profesor, los tiempos dedicados a la actividad y principales elementos del lenguaje favorecidos por la actividad y escala de apreciación conceptual, a efectos de emitir un juicio valorativo de la herramienta audiovisual utilizada los estudiantes participantes de la investigación contestaran en forma separada una encuesta de apreciación conceptual.

Algunos de los canales YouTube seleccionados por los estudiantes fueron:

ETJ English

(https://www.youtube.com/channel/ UCBHtnQGUChkwG1eyWL5EA9g)

- $\quad$ Amigos Ingleses

(https://www.youtube.com/channel/UCOJHr SvK4HsYnYLXCn4ifQ)

- $\quad$ How to learn More English with Emma. (https://www.engvid.com/how-to-learn-more-englishwith-emma/)

- How to learn Grammar, any Grammar. (https://www.youtube.com/watch?v=c6Y1UJb2fvc)

\author{
MmmmEnglish! \\ (https://www.youtube.com/channel/ \\ UCrRiVfHqBIlvSgKmgnSY66g) \\ English like a native. (https://www.youtube. \\ com/channel/UCOHg2Ks00kCekyjZG_LxOmg) \\ - Alejo Lopera Inglés
(https://www.youtube.com/watch?v=dDvu9Qal3js)
}

\section{POBLACIÓN Y MUESTRA.}

En esta investigación participaron 18 estudiantes distribuidos de la siguiente manera: 11 estudiantes de tercer nivel de la Carrera Pedagogía en Inglés, Universidad de La Serena y 07 estudiantes de Tercer Año Medio del Colegio José Manuel Balmaceda de la comuna de La Serena.

\section{DISCUSIÓN}

Las técnicas de recolección de información fueron: el formulario de adscripción a la investigación, el análisis documental de fichas de trabajo de los estudiantes del uso de la plataforma YouTube, denominadas en este trabajo Fichas de Capítulo y Focus Group.

El análisis e interpretación de los resultados se realizará mediante el método de triangulación de la información, entendida como una técnica donde se usan tres fuentes de datos de carácter cualitativo para verificar los objetivos propuestos en la investigación: formulario de adscripción, ficha de capítulo y Focus Group.

Respecto al formulario de adscripción al proyecto los estudiantes se inscribieron libremente $y$ seleccionaron un canal YouTube liberado, para su trabajo y aprendizaje autónomo. En este formulario consignaron los fundamentos de la elección del canal, sus expectativas de la actividad y las temáticas de aprendizaje más relevantes. Es importante señalar que la totalidad de los participantes señala que espera que el canal sea entretenido e interactivo. La mayoría manifiesta que tiene expectativas de mejorar la pronunciación, aprender nuevo vocabulario 
al igual que repasar aspectos gramaticales. Las temáticas más relevantes que buscan en el canal son: práctica de inglés en contexto, conversaciones en situaciones naturales y uso avanzado del idioma inglés.

La ficha de capítulo del estudiante tuvo como propósito sistematizar su aprendizaje de cada una de las clases presenciadas en la plataforma. En este instrumento los estudiantes consignaban: "Lo que vi me sirve para...", "Aplicabilidad de lo aprendido...", "Lo que más me gustaría saber de inglés..." El 100\% de los participantes del estudio siguió el protocolo señalado en este instrumento, lo que denotó rigurosidad en su trabajo, además de establecer jerarquía de las habilidades observadas en cada una de las sesiones estudiadas.

Tabla 1: Matriz para el análisis de ficha
Los estudiantes de enseñanza media elaboraron un total de 48 fichas de capitulo con un promedio de 7 por participante.

Los estudiantes de la Universidad elaboraron un total de 125 fichas de capitulo, alcanzando un promedio de 20 fichas por estudiante y un número similar de participaciones en clases de la plataforma.

Selección y organización de las fichas de acuerdo al nivel de dominio de habilidades trabajadas en las fichas- Taxonomía habilidades básicas, intermedias y avanzadas para la adquisición de la lengua, información que se organizó en la siguiente matriz.

\begin{tabular}{|c|c|c|}
\hline Habilidades de la Lengua & Nivel cognitivo & Contenidos \\
\hline Hablar & $\begin{array}{c}\text { Básicas } \\
\text { Intermedias } \\
\text { Avanzadas }\end{array}$ & \\
\hline Escuchar & $\begin{array}{c}\text { Básicas } \\
\text { Intermedias } \\
\text { Avanzadas }\end{array}$ & \\
\hline Leer & $\begin{array}{c}\text { Básicas } \\
\text { Intermedias } \\
\text { Avanzadas }\end{array}$ & \\
\hline Escribir & $\begin{array}{c}\text { Básicas } \\
\text { Intermedias } \\
\text { Avanzadas }\end{array}$ & \\
\hline
\end{tabular}

El trabajo sostenido del estudiante mediante el uso de la plataforma YouTube, tanto de la Universidad como del Colegio Municipal, a lo largo de cuatro meses, nos permitió detectar que el $100 \%$ de ellos subió sus niveles de motivación para el aprendizaje de la lengua, asumió la actividad con entusiasmo y sistematicidad. Al término de la investigación señalaron que habrían mejorado sus aprendizajes de las habilidades de la lengua inglesa, situación que se reflejó en mejores calificaciones al cierre del curso. Por otro lado, todos los estudiantes participantes del proyecto de investigación valoran positivamente la estrategia utilizada para el desarrollo de aprendizaje autónomo. 
Tabla 2: Matriz de análisis focus group estudiantes

\begin{tabular}{|c|c|c|c|c|}
\hline $\begin{array}{l}\text { Ejes de } \\
\text { conversación }\end{array}$ & Categoría & Subcategorías & $\begin{array}{l}\text { Constructos } \\
\text { relevantes }\end{array}$ & Registro textual \\
\hline $\begin{array}{l}\text { Valoración del } \\
\text { Canal } \\
\text { Youtube. }\end{array}$ & $\begin{array}{l}\text { Motivación para } \\
\text { selección del } \\
\text { canal. } \\
\text { Valoración de las } \\
\text { actividades para } \\
\text { el aprendizaje. }\end{array}$ & $\begin{array}{l}\text { Metodología } \\
\text { utilizada por el } \\
\text { recurso } \\
\text { audiovisual. } \\
\text { Tipo de } \\
\text { actividades. }\end{array}$ & $\begin{array}{l}\text { Presencia de una } \\
\text { metodología } \\
\text { activa. } \\
\text { Actividades que } \\
\text { contribuyeron en } \\
\text { forma efectiva a } \\
\text { su aprendizaje }\end{array}$ & $\begin{array}{l}\text { Respecto a la } \\
\text { metodología, en la } \\
\text { conversación, se puede } \\
\text { percibir que hay factores } \\
\text { que son muy bien } \\
\text { apreciados, Estudiante } 3 \\
\text { dice "el canal que } \\
\text { mencioné es el que captó } \\
\text { más mi atención, porque } \\
\text { más allá de subir } \\
\text { contenidos de gramática } \\
\text { el profesor se centraba } \\
\text { más en dar consejos. } \\
\text { Un buen sistema de } \\
\text { enseñanza de idioma } \\
\text { espera impactar en el } \\
\text { desarrollo de las cuatro } \\
\text { habilidades del lenguaje. } \\
\text { Estudiante } 2 \text { expresa; } \\
\text { "Yo creo que desarrollé } \\
\text { más la habilidad auditiva } \\
\text { y la parte de expresión } \\
\text { porque trataba de verlos } \\
\text { sin subtítulos y entender } \\
\text { lo que decía la profesora, } \\
\text { y si no lograba entender } \\
\text { ponía los subtítulos y ahí } \\
\text { escuchando a la vez." }\end{array}$ \\
\hline & $\begin{array}{l}\text { Actividades de } \\
\text { seguimiento y } \\
\text { evaluación }\end{array}$ & $\begin{array}{l}\text { Actividades de } \\
\text { evaluación }\end{array}$ & $\begin{array}{l}\text { Evaluación de los } \\
\text { temas expuestos. }\end{array}$ & $\begin{array}{l}\text { Estudiante } 8 \text { indica: "(mi } \\
\text { evaluación) era como un } \\
\text { quiz, uno entraba a una } \\
\text { página, por ejemplo, de } \\
\text { gramática, de acuerdo al } \\
\text { video, era con }\end{array}$ \\
\hline
\end{tabular}




\begin{tabular}{|c|c|c|c|c|}
\hline & $\begin{array}{l}\text { Impacto sobre } \\
\text { habilidades del } \\
\text { lenguaje }\end{array}$ & $\begin{array}{l}\text { Habilidades del } \\
\text { lenguaje } \\
\text { favorecidas }\end{array}$ & $\begin{array}{l}\text { Percepción sobre } \\
\text { impacto sobre el } \\
\text { desarrollo de } \\
\text { habilidades del } \\
\text { lenguaje. }\end{array}$ & $\begin{array}{l}\text { alternativas y uno tenía } \\
\text { que responder, todas con } \\
\text { alternativas que uno } \\
\text { seleccionaba y al final } \\
\text { salía el puntaje." } \\
\\
\text { Estudiante } 4 \text { dice: "Yo } \\
\text { creo que desarrollé más } \\
\text { la habilidad auditiva y la } \\
\text { parte de expresión } \\
\text { porque trataba de verlos } \\
\text { sin subtítulos y entender } \\
\text { lo que decía la profesora, } \\
\text { y si no lograba entender } \\
\text { ponía los subtítulos y ahí } \\
\text { iba leyendo y } \\
\text { escuchando a la vez." }\end{array}$ \\
\hline $\begin{array}{l}\text { Estrategias de } \\
\text { trabajo } \\
\text { autónomo. }\end{array}$ & $\begin{array}{l}\text { Registro de } \\
\text { trabajo } \\
\text { autónomo. }\end{array}$ & $\begin{array}{l}\text { Técnicas de trabajo } \\
\text { autónomo. }\end{array}$ & $\begin{array}{l}\text { Forma de registrar } \\
\text { la información. }\end{array}$ & $\begin{array}{l}\text { La presente investigación } \\
\text { considera un registro } \\
\text { metódico de la } \\
\text { información a la cual se } \\
\text { han expuesto } \\
\text { voluntariamente los } \\
\text { participantes: } \\
\text { Al respecto Estudiante } 3 \\
\text { dice: “Yo cuando veía un } \\
\text { capítulo, primero me } \\
\text { enfocaba en el título y } \\
\text { veía el tema porque en la } \\
\text { ficha salía tema y para } \\
\text { que me sirve, entonces } \\
\text { yo antes de hacer la ficha } \\
\text { veía el video y me } \\
\text { enfocaba en qué va a } \\
\text { tratar, mientras veía }\end{array}$ \\
\hline
\end{tabular}




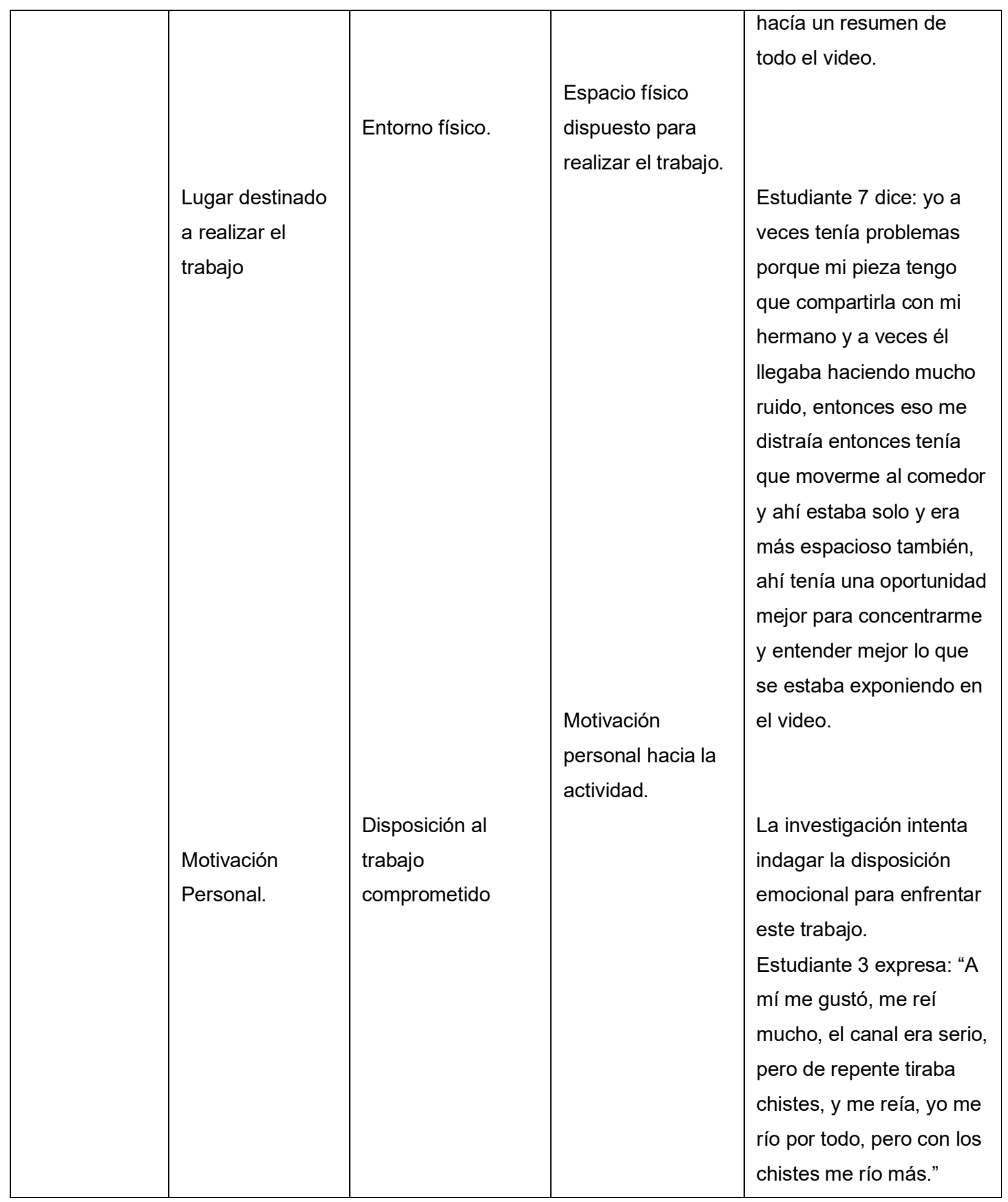


Los hallazgos relevados en el focus group nos muestran que los estudiantes, tanto universitarios como del colegio, valoran positivamente la estrategia audiovisual como lo es un canal de YouTube, en tanto estrategia para aprender. Los participantes tienen preferencia por el acento británico que se presenta en las clases de la plataforma. También se destaca que la presencia de una metodología activa, el uso de ejemplos vívidos, las pequeñas dramatizaciones como una forma directa de interactuar son destacadas por ellos. También valoran que el canal tenga actividades variadas desde la lectura hasta la gramática. Los participantes declaran que aprendieron en forma efectiva; vocabulario, expresiones idiomáticas, formas de hilar oraciones, gramática, pronunciación, escritura de párrafos, puntuación entre otros. En general no encontraron distractores importantes a excepción de algunos contenidos "muy básicos para su nivel".

Todos hicieron el trabajo con agrado e interés a pesar del poco tiempo disponible u otras distracciones. Mencionan, en general, pocas mejoras que harían a las actividades o al recurso didáctico con el cual trabajaron. Manifiestan opiniones divididas respecto de si una actividad como la realizada debería ser parte de su plan de formación formal. Además, manifiestan que el interés personal juega un rol muy importante ya que en esta investigación participaron por decisión personal y lealtad con el profesor convocante, además de verlo como una oportunidad de demostrar compromiso y deseos de mejorar sus rendimientos académicos. Finalmente, todos recomendarían un trabajo así, en algunos casos haciendo la salvedad que el compromiso y la motivación personal son muy importantes.

En síntesis, se puede decir que los estudiantes reconocen tener el hábito de registrar sistemáticamente lo que observan de la clase en la plataforma. Manifiestan que siguen una formalidad y método para realizar registros de información. Resaltan también, la interacción persona a persona que se logra en la acción de exponerse a una clase grabada y valoran la posibilidad de repetir partes de los contenidos que no comprenden fácilmente.

Los estudiantes le asignan una mucha importancia el lugar habitual de estudio para realizar la actividad de aprendizaje, su habitación. Destacan la posibilidad de portar los videos en su celular para verlos en cualquier lugar, auto, locomoción colectiva. Esto denota su alta disposición para el trabajo y realización de la tarea.

Es notable el nivel de compromiso de los estudiantes convocados para la investigación, todos lo asumieron como una tarea personal basada en la buena disposición al trabajo. Manifiestan una alta valoración del trato personalizado, las ayudas metodológicas, las pausas y ritmo de entrega de información en la clase virtual. Algunos expresan la necesidad de uso más frecuente de dramatización.

Los estudiantes participantes de la investigación recomiendan que este tipo de proyectos formen parte de las metodologías de la clase como un insumo de autoaprendizaje en la casa, la opinión es unánime, todos resaltan el hecho de que la clase Web es personalizada, eso no lo desean alterar.

Finalmente, la opinión unánime de los estudiantes, respecto de recomendar el trabajo realizado en el proyecto de investigación es compartida como una instancia efectiva para el aprendizaje del inglés.

\section{CONCLUSIONES.}

El trabajo autónomo del estudiante ha de ser un espacio para desarrollar el aprendizaje autónomo, como condición para continuar aprendiendo durante toda la vida, si bien se plantea dentro de los ideales formativos y dentro 
de los perfiles de egreso de los educandos, no parece tener un espacio propio en los currículos.

Finalmente es preciso señalar lo siguiente:

- El uso de la plataforma YouTube, con objetos de aprendizaje para desarrollar habilidades de la lengua inglesa en estudiantes de enseñanza media y educación superior, es un recurso altamente valorado, tanto por los docentes investigadores como por los estudiantes participantes de la investigación, dado que genera una interacción más horizontal y dialógica aun considerando que es un recurso virtual.

- Todos los participantes manifiestan que hicieron el trabajo con agrado e interés a pesar del poco tiempo disponible $u$ otras distracciones. Manifiestan que el interés personal juega un rol muy importante para trabajar con esta herramienta metodológica.

- Valoran positivamente la experiencia de participar en el proyecto y destacan la metodología de trabajo con la plataforma ya que se sienten involucrados por un trato directo a partir del lenguaje y actitud que adopta el docente que realiza la actividad formativa.

- Valoran el sentido del humor, como factor motivacional, que utiliza el docente al presentar las actividades de aprendizaje en la plataforma.

- Con respecto al tipo de aprendizaje que desarrollan con la plataforma valoran el uso de fichas para registrar lo aprendido como herramienta de avaluación formadora $y$ formativa, generando un ejercicio metacognitivo en el desarrollo de las habilidades comunicativas de la lengua inglesa.

\section{REFERENCIAS BIBLIOGRÁFICAS}

Consejo de Europa. (1992). Transparency and coherence in language learning in Europe: objectives, evaluation, certification, (informe editado por B. North basado en el simposio celebrado en Rüschlikon en 1991), Estrasburgo: Consejo de Europa.

Crispín, M.L. et al. (2011). Aprendizaje autónomo: orientaciones para la docencia. México: Universidad Iberoamericana, AC.

De Miguel, M. (2006). Métodos y Modalidades de enseñanza centradas en el desarrollo de competencias. Madrid: Alianza Universidad.

Harmer, J. (2001). English Language Teaching. Malaysia: Pearson Education Limited

Hernández, L. (200). Los métodos de enseñanza de las lenguas y las teorías de aprendizaje. Encuentro: revista de investigación e innovación en la clase de idiomas, 2000, n.11, p. [141]-153. ISSN 1130-7021.

Howatt, A. (1985). A history of English language teaching. Oxford: Oxford University Press.

Hymes, D. (1972). On Communicative Competence. In Pride, J. B., \& Holmes, J. (Eds.), Sociolinguistics, 269-293. Baltimore, USA: Penguin Education, Penguin Books Ltd.

Pintrich, P. R. and De Groot, E. V. (1990). Motivational and self-regulated learning components of classroom academic performance. Journal of Educational Psychology 82(1), 33-40

Pozo, J. y Monereo, C. (1999). El aprendizaje estratégico. Madrid, Aula XXI- Santillana.

Richards, J. \& Rodgers, S. (1986). Approaches and Methods in Language Teaching. A Description and Analysis. New York, USA: Cambridge University Press. 
Richards, J. (1997). Longman Dictionary of Language Teaching and Applied Linguistics. Londres: 7ma Ed. Longman.

Ruay, R y Garcés, J. (2015). Diseño y construcción de instrumentos de evaluación de aprendizajes y competencias. Colombia: Editorial Redipe.

Ruiz, José María1996). ¿Cómo hacer una evaluación de centros educativos? Madrid: Narcea S.A de Ediciones.

Stake, R. (1999). Investigación con estudio de casos. Madrid: Ediciones Morata

Tamayo y Tamayo, M. (2002). El proceso de investigación científica. Cuarta Edición. México: Editorial Limusa. S.A.

Wilkings, D. (1972). Grammatical, Situational and Notional Syllabuses. Proceedings of the Third International Congress of Applied Linguistics. Copenhague: Julius Groos 